\title{
Genome-wide Identification and Transcriptional Characterization of Dna Methyltransferases Conferring Temperature-sensitive Male Sterility in Wheat
}

\section{Dan Li}

Beijing Academy of Agriculture and Forestry Sciences https://orcid.org/0000-0001-5693-1056

\section{Bian-e Feng}

Shanxi Agricultural University

\section{Yong-Jie Liu}

Beijing Academy of Agriculture and Forestry Sciences Jie Gong

Beijing Academy of Agriculture and Forestry Sciences

\section{Yi-Miao Tang}

Beijing Academy of Agriculture and Forestry Sciences

\section{Li-Ping Zhang}

Beijing Academy of Agriculture and Forestry Sciences

\section{Bin-Shuang Pang}

Beijing Academy of Agriculture and Forestry Sciences

\section{Ren-Wei Sun}

Beijing Academy of Agriculture and Forestry Sciences

\section{Feng-Ting Zhang}

Beijing Academy of Agriculture and Forestry Sciences

\section{Zhao-Bo Chen}

Beijing Academy of Agriculture and Forestry Sciences

\section{Yong-Bo Wang}

Beijing Academy of Agriculture and Forestry Sciences

\section{Xian-Chao Chen}

Beijing Academy of Agriculture and Forestry Sciences

\section{Ai-Ping Wang}

Shanxi Agricultural University

\section{Chang-Ping Zhao}

Beijing Academy of Agriculture and Forestry Sciences

Shiqing Gao ( $\sim$ gshiq@126.com ) 
The Municipal Key Laboratory of the Molecular Genetics of Hybrid Wheat, Beijing Engineering Research Center for Hybrid Wheat, Beijing Academy of Agriculture and Forestry Sciences, Beijing 100097, China https://orcid.org/0000-0003-0348-9668

\section{Research article}

Keywords: Triticum aestivum L., temperature-sensitive genic male sterility (TGMS), stress response, DNA methyltransferases, transcript abundance

Posted Date: October 7th, 2020

DOI: https://doi.org/10.21203/rs.3.rs-81486/v1

License: (1) This work is licensed under a Creative Commons Attribution 4.0 International License. Read Full License

Version of Record: A version of this preprint was published at BMC Genomics on April 29th, 2021. See the published version at https://doi.org/10.1186/s12864-021-07600-7. 


\section{Abstract}

Background: DNA methyltransferase (DMT) genes contribute to plant stress responses and development by de novo establishment and subsequent maintenance of DNA methylation during replication. The photoperiod or/and temperature-sensitive genic male sterile (P/TGMS) lines play an important role in hybrid seed production of wheat. However, only few studies have been reported about the effect of the DMT genes on temperature-sensitive male sterility of wheat. Although DMT genes have been researched in some plant species, the identification and analysis of DMT genes in wheat (Triticum aestivum L.) based on genome-wide levels has not been reported.

Results: In this study, a detailed overview of phylogeny of 52 wheat DMT (TaDMT) genes was presented. Homoeolog retention for TaDMT genes was significantly above the average retention rate for wholewheat genes, indicating the functional importance of many DMT homoeologs. We found that the strikingly high number of TaDMT genes mainly resulted from the significant expansion of TaDRM subfamily. Intriguingly, all 5 paralogs belonged to the wheat DRM subfamily and we speculated tandem duplications might play a crucial role in the TaDRM subfamily expansion. Through the transcriptional analysis of TaDMT genes in a TGMS line BS366 and its hybrids and its hybrids with other six fertile lines under sterile and fertile conditions, we concluded that TaCMT-D2, TaMET1-B1, and TaDRM-U6 might be involved in male sterility in BS366. Furthermore, a correlation analysis showed that TaMET1-B1 might negatively regulate the expression of TaRAFTIN1A, an important gene for pollen development, so we speculated an epigenetic regulatory mechanism underling the male sterility of BS366 by the interaction between TaMET1-B1 and TaRAFTIN1A.

Conclusions: Our findings presented a detailed phylogenic overview of the DMT genes and could provide novel insights into the effects of the DMT genes on TGMS wheat.

\section{Background}

DNA methylation is a heritable epigenetic modification widely found in bacteria, animals and plants and instrumental to transposable elements (TEs) suppression, gene silencing, genomic imprinting and chromosome inactivation $[1,2]$. DNA methylation is catalyzed by DNA methyltransferase (DMT) that introduce a methyl group at the $\mathrm{C} 5$ or $\mathrm{N} 4$ position of cytosine or the N6 position of adenine. C5-cytosine DNA methylation (simply named here as DNA methylation) is the most prevalent among all these modifications, especially in the plant [3,4]. DNA methylation occurs in multiple sequence contexts, including $\mathrm{CG}$ and $\mathrm{CHG}$, both of which are symmetric, and $\mathrm{CHH}$ which is asymmetric $(\mathrm{H}=\mathrm{A}, \mathrm{T}$, or $\mathrm{C})[5,6]$. It has been found that there are two major methods modulating DNA methylation, including de novo methylation and maintenance methylation [7, 8]. Maintenance methylation occurs during DNA replication on hemimethylated CG and CHG contexts. De novo methylation occurs at all three unmethylated cytosines $[3,9]$. 
In plants, DNA methylation is controlled by four subfamilies of DMT genes: Methyltransferase 1 (MET1, the homologs of mammalian DNMT1), Chromomethylase (CMT, plant-specific), Domains Rearranged Methyltransferase (DRM, the homologs of mammalian DNMT3) and DNA methyltransferase 2 (DNMT2) [10-12]. The MET1 genes play a pivotal role in maintaining methylation in CG context. Arabidopsis thaliana harbored four MET1 homologs, but only the loss-of-function of MET1 bored phenotypic consequences. Ablation of MET1 resulted in nearly all loss of CG methylation in Arabidopsis thaliana [13-15]. In rice (Oryza sativa L.), there are two MET1 genes (OSMET1A and OsMET1B), only OsMET1B (OSMET1-2) plays an essential part in maintaining CG methylation during normal growth and development. A null mutation of OSMET1B leaded to genome-wide hypomethylation [16]; however, knockout of OSMET1A (OSMET1-1) didn't show alteration of DNA methylation at assessed marker loci [17]. The maintenance of major $\mathrm{CHG}$ methylation is accomplished by CMT3 [18]. There are three CMT genes (AtCMT1, AtCMT2, and AtCMT3) in Arabidopsis thaliana and AtCMT3 is the main CHG methyltransferase. In the mutant of AtCMT3, a strong depletion of CHG methylation was observed [19]. In addition, previous research showed that CMT2 could partly partake in the maintenance of $\mathrm{CHH}$ methylation via cross-talk with histone modifications in plants $[11,12]$. The DRMs are primarily responsible for catalyzing de novo DNA methylation in all three contexts achieved by RNA-directed DNA methylation (RdDM) pathway in plant. RdDM involves two specific RNA polymerases, RNA Pol IV, and Pol $\mathrm{V}$, as well as $24 \mathrm{nt}$ small interfering RNAs (siRNAs) [20-23]. DRM2 is required for de novo DNA methylation of all three contexts and for the maintenance of non-CG methylation [24, 25]. After the targeted destruction of OSDRM2, the CG and non-CG methylation levels were reduced and de novo methylation mediated by RdDM process was deficient [26]. Based on sequence homology, the DNMT2 was originally considered to be DNA methyltransferase. However, robust DNA methyltransferase activity could not be observed using DNMT2 preparations and the considerable variability in the target DNA sequences showed that DNMT2 enzymes were actually tRNA transferases [27-29].

Comprehensive studies have showed that plant DNA methylation was predominantly associated with growth and development [30,31], secondary metabolism [32,33] and various stress responses including salt stress [34,35], drought stress [36, 37], cold stress [38, 39]. Since DNA methylation is involved in regulating a wide range of biological processes, DMT genes have been identified and analyzed in several plant species, such as Arabidopsis thaliana [40, 41], rice [42, 43], maize [44, 45], and wheat [46]. However, to the best of our knowledge, no investigator has focused on the identification and analysis of DMT genes in wheat at a whole-genome level.

Wheat (Triticum aestivum L.) is one of the most important staple crops worldwide, contributing a significant amount of proteins and calories to the global human population [47, 48]. Given a growing world population coupled with increasingly challenging cultivation conditions, facilitating breeding and large-scale adoption of hybrid wheat are of great importance to the food supply $[49,50]$. The two-line system based on photoperiod or/and temperature-sensitive genic male sterile (P/TGMS) line plays an important role in hybrid seed production of wheat [51]. Male sterility of the P/TGMS line is heritable, but regulated by an appropriate temperature or photoperiod. In TGMS line of wheat, the pollen viability is dramatically decreased to complete sterility at a certain low temperature occurring during reproductive 
development $[52,53]$. Temperature stress may lead to complete sterility and severe losses in grain yield during reproductive development, mainly because the reproductive stage is especially sensitive to environmental stresses [54]. Particularly, male reproductive organs are known to be more sensitive to temperature stress than female organs in higher plants [55]. Plants have developed diverse mechanisms to survive extreme environmental conditions. Changes in DNA methylation have been shown to play a crucial part in plant stress responses [6]. Additionally, some recent reports revealed that DNA methylation levels are affected by DMTs and regulated the expression of genes involved in cytoplasmic male sterile in rice, maize, and wheat [56-58]. However, few studies have been reported about exploring the roles of DMTs in TGMS wheat during the reproductive stage.

To better understand the dynamic evolution of DMT gene in wheat and to facilitate further research on this important gene family, all members of DMT genes in wheat were identified; their phylogenetic relationships and gene structures were investigated. We found wheat DRM subfamily significantly larger than expected and hypothesize that high homoeolog retention in the DRM subfamily contributed to the expansion of wheat DMT genes. Moreover, the transcript abundance of DMT genes under sterile and fertile conditions was analyzed. Notably, we found that most TaDMT genes were significantly upregulated under sterile conditions compared to fertile conditions. After further expressional analysis, we concluded that TaCMT-D2, TaMET1-B1, and TaDRM-U6 were more likely associated with male sterility in BS366, particularly, the interaction between TaMET1-B1 and TaRAFTIN1A. The results of this study present a detailed overview of phylogeny of the DMT genes and provide novel insights into the functional roles of the DMT genes in male sterility of TGMS wheat.

\section{Results}

\section{Identification and phylogenetic analysis of DMT genes}

In order to analyze the function of DMT genes, we performed genome-wide identification and phylogenetic analysis of DMT genes in wheat. A total of 52 DMT genes were identified on the basis of the functional annotation (Pfam domains) in the IWGSC archive v.1.0 [59]. We named all TaDMT genes according to their subfamily association and subgenome location (Supplemental Table S1). Arabidopsis and rice have 10 and 7 DMTs, respectively (Fig. 1). Wheat has the largest DMT genes in the three species; this is partly the result of the hexaploid nature of wheat. However, even corrected for ploidy level, the number of DMT genes in wheat was significantly higher than in rice (c. 2.5 -fold higher; $\chi^{2}$ test, $P=0.02$ ). The increase in number of TaDMT genes is mainly a result of the gene counts in the TaDRM subfamily that is significantly larger than expected $\left(X^{2}\right.$ test, $\left.P=0.01\right)$. The numbers of genes in the remaining subfamilies were not significantly different from the expected 3:1 ratio.

To elucidate the phylogenetic relationship of DMT genes from Arabidopsis thaliana, rice (Oryza sativa L.), and wheat, a total of 69 DMT protein sequences were used. A maximum likelihood phylogenetic tree of DMT genes shows that the TaDMT genes can be naturally grouped into four subfamily: MET1, CMT, DRM, and DNMT2 (Fig. 1). In all subclades, the DMT phylogeny roughly followed species phylogeny. As 
the Arabidopsis genes is a sistergroup related to the grass genes, OsDMT genes were more closely related to a triad of wheat homologs such as in the MET1 and CMT subfamily (Fig. 1).

\section{Chromosomal location and homoeologous genes of DMT genes in wheat genome}

DMT genes unevenly distributed across wheat chromosomes: chr1A, chr1B, and chr1D had no TaDMT gene; the highest number of genes are located on chr4B (7 genes) (Fig. 2). Interestingly, different members of DMT gene subfamilies are located in chromosomal regions that might represent homoeologous segments resulting from ancestral events [49]. About 69\% (12 triads) of the 52 DMT genes identified are present in triads. Additionally, 23\% (six pairs) of DMT genes lost one homoeologou gene of the triads in wheat genome. Thus, the high homoeologous retention rate can partly explain the high number of wheat DMT genes. Intriguingly, there were 5 paralogs in the wheat genome that were all from the DRM subfamily, pointing that tandem duplication might an important mechanism for subfamily expansion (Fig. 2).

\section{Conserved motifs and gene structures of DMTs in wheat}

Phylogenetic relationships of wheat DMTs showed that the MET1 and CMT subfamily were more similar and belonged to the same clade (Fig. 3a). Furthermore, the motifs in all members of TaDMT genes were analyzed. A total of 10 different motifs were identified in 52 TaDMT proteins (Fig. 3b). Among the motifs, motif 1, 3, 8, and 9 were found in all TaCMT and TaMET1, Hence, these two subfamily proteins were clustered together to be a small branch. TaDNMT2 was the smallest subfamily in the wheat phylogenetic tree and contained only 3 genes. The TaDNMT2 proteins included motifs 3 and 8 . Most TaDRM genes were composed of 8 motifs, including in sequential order motifs 10, 5, 2, 6, 3, 4, 7, and 1, which has a large difference from the structure of the other three TaDMT genes. This finding is consistent with the results of the gene structures of TaDMT genes (Fig. 3c). The TaDMT genes exhibited different exon-intron organizational patterns. TaCMT genes had the largest number of exons; however TaDRM genes had the least number of exons.

DMT genes were significantly enriched in thermosensitive genic male sterile line BS366 under sterile and fertile conditions

A published GeneChip ${ }^{\circledR}$ data set was used to seek for differently expressed genes (DEGs) under sterile and fertile conditions in temperature-sensitive genic male sterile line BS366 anthers during meiosis [52]. The up- and down- regulated probe sets were used to enrichment analysis by Gene Ontology (GO). The top enriched biological process GO terms included DNA methylation (Fig. 4a), indicating that DNA methylation might play an important role in affecting fertility in wheat. In order to further understand the function of DNA methylation, we first analyze the function of DMT genes in wheat and the expressed transcripts of DMT genes in BS366. 
To clarify the potential roles of TaDMT genes involved in male sterility, we analyzed transcript abundance of TaDMT genes in BS366 based on the GeneChip ${ }^{\circledR}$ data. In this study, there were 13 TaDMT genes expressed and 10 of them were differentially expressed under sterile and fertile conditions (Fig. 4b). 9 TaDMT genes were up-regulated and only TaMET1-D1 was down-regulated under sterile conditions compared to fertile conditions. TaMET1-B1 and TaCMT-D2 were significantly differentially expressed in $1.5 \mathrm{~mm}$ anthers stage; TaMET1-D1 were significantly differentially expressed in $2.0 \mathrm{~mm}$ anthers stage; TaMET1-B1, TaCMT-A4, TaDRM-B7, TaMET1-B2, TaMET1-B3, TaCMT-B5, and TaCMT-D2 were significantly differentially expressed in $2.2 \mathrm{~mm}$ anthers stage; TaMET1-B1, TaMET1-B2, TaCMT-D2, $T a D R M-D 9$, and TaDRM-U6 were significantly differentially expressed in $2.5 \mathrm{~mm}$ anthers stage. TaMET1B1, TaDRM-B7, TaMET1-B2, TaMET1-B3, TaCMT-B5, TaDRM-D9, and TaDRM-U6 were specifically differentially expressed in $3.0 \mathrm{~mm}$ anthers stage. Almost all differential expression of TaDMT genes occurred during 2.2, 2.5, or $3.0 \mathrm{~mm}$ anthers stage. From the $2.2 \mathrm{~mm}$ anthers stage mitosis ceased and transited to meiosis I according to morphological observations. The result reinforced the conclusion that cold stress contributed to aberrant cytokinesis during male meiosis I in BS366 [52]. Therefore, it was speculated that $2.2 \mathrm{~mm}, 2.5 \mathrm{~mm}$, and $3.0 \mathrm{~mm}$ periods are the key periods affecting male sterility in BS366. So, we chose TaMET1-B1, TaCMT-A4, TaDRM-B7, TaMET1-B2, TaMET1-B3, TaCMT-B5, TaCMT-D2, TaDRM-D9, and TaDRM-U6 to further assess their expression.

\section{Transcript abundance of TaDMT genes in fertile lines and hybrids of wheat under sterile and fertile conditions}

To verify the expression of 9 TaDMT genes involved in male fertility in BS366, the anthers sampled from BS366, four fertile lines (J411, TY806, MC159, and GLDS) and three hybrids (GLDS × BS366, MC159 × BS366, and TY806 $\times$ BS366) at the meiosis stage were used. TaCMT-D2, TaCMT-B5, TaMET1-B1, TaMET1-B2, TaMET1-B3, TaDRM-U6, TaDRM-B7, and TaDRM-D9 were confirmed up-regulated in BS366 under sterile compared to fertile condition and down-regulated or not differently expressed in fertile lines (Fig. 5). TaCMT-A4 expressed no difference in BS366 while expressed differently in J44 and MC159 under sterile compared to fertile condition (Fig. 5b). BS366 and three fertile lines (TY806, MC159, and GLDS) have been used to generate three different fertility-restored hybrids: TY806 × BS366 (91.17\%), MC159 × BS366 (90.60\%), and GLDS × BS366 (89.83\%) [60]. Given this, TY806, MC159, GLDS, and their hybrids with BS366 were considered excellent materials to assess the potential association of TaDMT genes with male sterility in BS366. Up-regulated expression were detected for TaCMT-D2, TaCMT-B5, TaMET1-B1, TaMET1-B2, TaMET1-B3, TaDRM-U6, TaDRM-B7, and TaDRM-D9 in GLDS $\times$ BS366, while down-regulated or not different expression were detected in TY806 $\times$ BS366 and MC159 $\times$ BS366 under fertile and sterile conditions (Fig. 5). Based on the above results, we speculated TaCMT-D2, TaCMT-B5, TaMET1-B1, TaMET1-B2, TaMET1-B3, TaDRM-U6, TaDRM-B7 and TaDRM-D9 might associate with male sterility in BS366.

To validate our speculation, we further analyzed the tissue-specific expression patterns of the eight TaDMT genes in roots, stems, leaves, and anthers using another three different fertility-restored $F_{1}$ hybrids 07GF83 × BS366 (97\%), 07YH91-43 × BS366 (96\%), and 7P395 × BS366 (89\%) under sterile 
conditions (Fig. 6b and 6d ). They were crossed by high-(07GF83), medium-(07YH91-43), and low-restorer lines (7P395) with BS366. Likewise, they were also prominent materials to evaluate the underlying association TaDMT genes with male sterility in BS366. In 07GF83 $\times$ BS366, the expression levels of TaCMT-D2, TaCMT-B5, TaMET1-B1, TaMET1-B2, TaDRM-U6, TaDRM-B7, and TaDRM-D9 in leaves were much higher under sterile compared to fertile conditions (Fig. 7a-7C). In 07YH91-43 × BS366, TaCMT-D2, TaMET1-B1, and TaDRM-U6 expressed more higher under sterile compared to fertile conditions in leaves; TaMET1-B3 expressed much higher under sterile compared to fertile conditions in anthers (Fig. 7d-7f). In 7P395 × BS366, TaCMT-D2, TaMET1-B1, TaMET1-B3, and TaDRM-U6 expressed higher under sterile compared to fertile conditions in anthers (Fig. 7g-7h). Take into account different fertility of 07GF83 $\times$ BS366, 07YH91-43 × BS366, and 7P395 × BS366, TaCMT-D2, TaMET1-B1 and TaDRM-U6 tissuespecifically differentially expressed in anthers in low fertility line 7P395 $\times$ BS366. So we concluded TaCMT-D2, TaMET1-B1, and TaDRM-U6 might take part in male sterility in BS366.

Expressional correlation analysis among candidate TaDMT genes and the other DEGs in BS366 under fertile and sterile conditions

To search genes that might interact with the candidate TaDMT genes, correlation analysis of expressional levels among the DEGs in BS366 under fertile and sterile conditions were conducted. The degree of correlation varied substantially among the genes and Fig. 8 showed the relative highly negative correlated genes with the candidate TaDMT genes. TaRAFTIN1A, an important gene for pollen development [61] and TraesCS4A02G093100 were most highly negative correlated with TaMET1-B1 $(\mathrm{r}=-0.99$, Fig. 8). We assume that the interaction between TaMET1-B1 and TaRAFTIN1A might play an important role in the male sterility of BS366. In addition, TaRAFTIN1A also has a relatively high negative correlation with TaDRM-U6 ( $\mathrm{r}=-0.94$, Fig. 8). The expressional pattern of TaRAFTIN1A in the anthers of BS366 and three hybrids (07GF83 $\times$ BS366, 07YH91-43 $\times$ BS366, and 7P395 $\times$ BS366) under fertile and sterile conditions were verified (Supplemental Fig. S2). Moreover, we analyzed the cis-acting elements in the promoter sequences of TaCMT-D2, TaMET1-B1, TaDRM-U6, and TaRAFTIN1A (Supplemental Fig. S3). According to the prediction of cis-acting elements, TaCMT-D2, TaMET1-B1, and TaRAFTIN1A genes contained the LTR element which was considered in response to low temperature.

\section{Discussion}

\section{Structural and evolutionary features of DMT genes in wheat}

The DMTs play an important role in modulating DNA methylation and gene expressional levels, which are involved in various stress responses [6]. They are therefore promising targets for crop breeding and improvement. In this study, a total of 52 DMT genes were identified in wheat (Supplemental Table S1;

Fig. 1). The number of DMT genes discovered in wheat is higher than that of in Arabidopsis (10) and rice (7). The number of DMT genes is 7.4 times higher in wheat than in rice. Leaving hexaploidy aside, the number of DMT genes in wheat was significantly higher than in rice (c. 2.5 -fold higher; $\chi 2$ test, $P=0.02$ ). Further, we found that the strikingly high number of TaDMT genes is mainly a result of the significant 
expansion of genes in TaDRM subfamily ( $\chi 2$ test, $P=0.01$ ). DNA methylation has long been thought to act mostly on control of repetitive elements within genomes [62]. Insertion and deletion of repetitive elements such as transposable elements (TEs) strongly impact genome size and evolution [63]. TEs may spread rapidly resulting in deleterious or null mutations within genomes, which presents a threat to genome stability and integrity resulting in plant structural and developmental defects [64]. To prevent this, DNA methylation mechanisms can be triggered by various mechanisms [65]. DNA methylation was considered as one of the key components in understanding divergence in genome evolution among plants [66]. Expansion of wheat DMT genes might contribute to its large genome size. In all, $69 \%$ (12 triads) of wheat DMT genes could be assigned to $1: 1: 1$ homoeologous groups (Fig. 2). This is significantly above the average homoeologous retention rate (35.8\%) in wheat [49]. Thus, the high homoeologous retention rate can partly explain the high number of wheat DMT genes. What's more, there were 5 paralogs in wheat genome that were all from DRM subfamily. Hence, we deduced that tandem duplication and high homoeologous retention rate may be the key factors in the evolution of DMT genes in wheat.

In phylogenetic classification, DMT genes were assigned to 4 conserved subfamilies, namely, MET1, CMT, DRM, and DNMT2. Except for the DRM subfamily, the numbers of genes in the remaining subfamilies were not significantly different from the expected 3:1 ratio factoring in ploidy level. In all subclades, the DMT phylogeny roughly followed species phylogeny and OsDMT genes were more closely related to the triad of wheat homologs. DMT genes diverged prior to the split of monocots and dicots, so we speculated that the functions of DMT genes may be different between dicots and monocots.

Although the number of DMT genes varied widely among different plant species, we found that they had conserved domains (Supplemental Fig. S1). All members of the DMT genes identified in this study contained the DNA-methylase domain, indicating it may be the core domain of DMT genes. Some studies have shown that the absence of DNA-methylase domain makes the DMT proteins can't normally methylate the cytosine [6, 67]. All members of TaDRM (27) and TaDNMT2 (3) only contained the DNAmethylase domain. The BAH domain (Bromo Adjacent Homology) existed in almost members of TaMET1 and TaCMT. MET1 and CMT3 mainly maintain CG and CHG methylation, separately [5], so we speculated the $\mathrm{BAH}$ domain plays a key role in the maintenance of symmetrical methylation. Indeed, it has been reported that when the BAH domain was disrupted in AtCMT1, the mutated AtCMT1 protein functioned abnormally in methylation maintenance in Arabidopsis [68]. Similarly, ablation of the BAH domains within DNMT1 (MET1) locus, the internally deleted protein lost the ability to mediate maintenance methylation during $S$ phase in embryonic stem (ES) cells [69]. Furthermore, almost members of TaMET1 and TaCMT also contained extra chromo and DNMT1-RFD domain, respectively. We speculate that the chromo and DNMT1-RFD domain are responsible for the recognition of hemi-methylated CHG and CG dinucleotides, separately.

\section{DMT genes play important roles in temperature-sensitive male sterile wheat}


Temperature and drought are the major abiotic stresses during reproductive stage that threat the growth and development of wheat. Particularly, male reproductive organs are known to be more sensitive to temperature stress than female organs in higher plants $[55,70]$. Temperature stress may lead to complete sterility during reproductive development [54]. The two-line system taking advantage of photoperiod or/and temperature-sensitive genic male sterile (P/TGMS) line plays a great role in hybrid seed production of wheat [51]. In TGMS line of wheat, the pollen viability is dramatically decreased to complete sterility at a certain low temperature occurring during reproductive development $[52,53]$. Recent studies have shown that DMT genes contribute to plant stress responses and development by de novo establishment and subsequent maintenance of DNA methylation [71]. However, the transcript abundance of DMT genes in temperature-sensitive genic male sterile wheat has rarely been studied.

In this study, we investigated the transcript abundance of the TaDMT genes in temperature-sensitive genic male sterile line BS366 based on the published GeneChip ${ }^{\circledR}$ data under sterile and fertile conditions. The results showed that there were 13 TaDMT genes expressed and 10 of them were differentially expressed in BS366 under sterile and fertile conditions. Almost the differentially expressed TaDMT genes were up-regulated and nearly all differential expression of TaDMT genes occurred during 2.2, 2.5 or $3.0 \mathrm{~mm}$ anthers stage (Fig. 4b). Therefore, it was speculated that $2.2 \mathrm{~mm}, 2.5 \mathrm{~mm}$ and $3.0 \mathrm{~mm}$ periods are the key periods affecting male sterility in BS366. We further analyzed and compared the expression levels of the 9 DMT genes in three fertile wheat lines and their different fertility-restored hybrids. We found up-regulated expression were detected for TaCMT-D2, TaCMT-B5, TaMET1-B1, TaMET1-B2, TaMET1-B3, TaDRM-U6, TaDRM-B7, and TaDRM-D9 in the low fertility-restored hybrid GLDS $\times$ BS366, while down-regulated or no different expression were detected in TY806 $\times$ BS366 (high fertility-restored hybrid) and MC159 × BS366 (medium fertility-restored hybrid) under fertile and sterile conditions. Then the tissue-specific expression patterns of the eight TaDMT genes were conducted using another three different fertility-restored hybrids. After analysis of the transcript abundance of TaDMT genes step by step under sterile and fertile conditions, we have an conclusion that TaCMT-D2, TaMET1-B1, and TaDRMU6 were the candidate TaDMT genes that might participate in male sterility in BS366.

To search target genes of the three candidate TaDMT genes, correlation analysis of expressional levels among the DEGs in BS366 under fertile and sterile conditions were conducted. We found that TaRAFTIN1A, an important gene for pollen development was highly negative correlated with TaMET1-B1 $(r=-0.99)$. We further speculate that the interaction between TaMET1-B1 and TaRAFTIN1A might play an important role in the male sterility of BS366. TaMET1-A1, TaMET1-B1, and TaMET1-D1 are the three wheat homoeologs of AtMET1 in Arabidopsis and OSMET1B in rice. Previous studies have shown that only the loss-of-function mutation of ATMET1 resulted in almost all loss of CG methylation in Arabidopsis thaliana $[13,14]$. Likewise, in rice, a null mutation of OsMET1B leads to genome-wide hypomethylation [16]; however, knockout mutant of OSMET1A (OSMET1-1) didn't show alteration of DNA methylation at assessed marker loci [17]. These results suggest the importance of TaMET1-A1, TaMET1B1, and TaMET1-D1 in the maintenance of CG methylation in wheat. In this study, TaMET1-A1 did not express in BS366; TaMET1-B1 was up-regulated; while TaMET1-D1 was down-regulated in BS366 under sterile condition compared to fertile conditions. This phenomenon has also been found in the MIKC-type 
MADS-box genes [49]. The number of balanced transcript abundance from all three homoeologs of MIKCtype MADS-box gene triads was relatively low compared with an average assessment of all wheat genes [49]. The unbalanced homoeolog expression pattern might predict different functions of different homoeolog alleles.

\section{Conclusions}

In this study, DNA methyltransferase (DMT) genes in wheat (Triticum aestivum L.) based on genome-wide levels was identified and transcriptional characterized to assess the regulation of DMT genes in the fertility of temperature-sensitive genic male sterile (TGMS) wheat BS366. We presented a detailed phylogenic overview of wheat DMT (TaDMT) genes. Homoeolog retention for TaDMT genes was significantly above the average retention rate for whole-wheat genes. We also found extensive expansion of TaDRM subfamily and tandem duplications might play a crucial role in the expansion of TaDRM subfamily. Through the transcriptional analysis of TaDMT genes in a TGMS line BS366 and its hybrids with other six fertile lines under sterile and fertile conditions, we concluded that TaCMT-D2, TaMET1-B1, and TaDRM-U6 might be involved in the male sterility of BS366. Moreover, a correlation analysis showed that TaMET1-B1 might negatively regulate the expression of TaRAFTIN1A, an important gene for pollen development. Our findings could provide novel insights into the effects of the DMT genes on TGMS wheat.

\section{Methods}

\section{Genome-wide identification of DMT genes}

Functional annotations of wheat, rice, and Arabidopsis genes were downloaded from the Ensembl (http://plants.ensembl.org/info/website/ftp/index.html). A hidden Markov model (HMM) analysis was performed in Protein family database (Pfam) to extract HMM profiles for the DNA methylase domain (PF00145), Chromo domain (PF00385) $\square$ BAH domain (PF01426) $D$ DNMT1-RFD domain (PF12047) from all species. The software hmmer was used to search for the DMT genes $(E<1 \mathrm{e}-10)$. Subsequently, Pfam and Conserved Domain Database (CDD, https://www.ncbi.nlm.nih.gov/cdd/) were used to confirm all the DMT genes contain the conserved domain. A total of 75 sequences were identified in wheat genome. After excluding splice variants and keeping only the first variant, 52 genes were used for further analysis.

\section{Maximum likelihood phylogeny, conserved motif, gene structures, and and cis-acting elements of DMT genes}

Related DMT sequences from wheat, rice, and Arabidopsis were aligned by Clustal W and then a phylogenetic tree was inferred using MEGA X [72] software by maximum likelihood method (bootstrap value $=1,000$ ). The motifs of DMT genes in wheat were analyzed with MEME database (http://memesuite.org/tools/meme), Parameter settings were -nmotifs 10 and the remaining parameters were the default. Based on genetic feature format data from wheat genome, the gene structures of DMT genes 
were analyzed using the Tbtools software [73]. To analyze the cis-acting elements of TaCMT-D2, TaMET1B1, TaDRM-U6, and TaRAFTIN1A, the upstream sequences (2,000 bp) of the start codon were retrieved from the wheat genome database, and then the cis-acting elements were analyzed by using the PlantCARE tool [74].

\section{Naming of DMT genes in wheat}

Naming wheat DMT genes, genome location and phylogenetic relationships were taken into account. The name of each gene started with Ta, an abbreviation for Triticum aestivum and followed by the name of Arabidopsis gene from this subfamily. And the subsequent name was $A, B$ or $D$, indicating the subgenome DMTs located in. Homoeologs were identified by phylogeny. Putative homoeologs have identical gene names except for the subgenome identifier (e.g. TaMET1-A1, TaMET1-B1, and TaMET1-D1). Genes belonging to the same subfamily but different triads within the same subgenome were consecutively numbered (e.g. TaCMT-A1 and TaCMT-A2). Paralogs were distinguished by consecutive numbers separated by a dash (e.g. TaDRM-A1-1 and DRM-A1-2).

\section{Plant materials, RNA isolation, and RT-PCR}

Wheat (Triticum aestivum L.) cv. BS366, Jing411, GLDS, TY806, MC159, and hybrids GLDS $\times$ BS366, MC159 × BS366, and TY806 × BS366, 07YH91-43 × BS366, 07GF83 $\times$ BS366, and 7P395 × BS366 were used for reverse transcription polymerase chain reaction (RT-PCR) analysis. Plants were grown according to Tang et al. [52] and at meiophase respectively treated for sterility treatment: $10^{\circ} \mathrm{C}$ with a $12 / 12-\mathrm{h}$ light/dark cycle or fertility treatment: $20^{\circ} \mathrm{C}$ with a 12/12-h light/dark cycle for 5 days. The anthers of Jing411, BS366, GLDS, TY806, MC159 GLDS $\times$ BS366, MC159 × BS366, and TY806 $\times$ BS366 and the roots, stems, leaves, and anthers of 07YH91-43 $\times$ BS366, 07GF83 $\times$ BS366, and 7P395 $\times$ BS366 were then collected and snap-frozen at $-80^{\circ} \mathrm{C}$. Total RNA was extracted using TRIzol reagent (Invitrogen, Carlsbad, CA, USA) according to the manufacturer's instructions. First-strand cDNA was synthesized using a Takara PrimeScript ${ }^{\text {TM }}$ RT Reagent Kit (Takara Biotechnology [Dalian] Co., Ltd., Dalian, China) using a random primer. An Eco Real-Time PCR System (Illumina, San Diego, CA, USA) with Takara SYBR® Premix Ex Taq ${ }^{\text {TM }}$ (Tli RNase H Plus; Takara Biotechnology [Dalian] Co., Ltd.) was used for RT-PCR. Actin acted as an internal control for the wheat expression studies. Primers were designed using Premier 3.0 (v. 0.4.0) (Supplemental Table S2). Relative gene expression levels were calculated using the $2^{-\triangle \Delta C T}$ method [75].

\section{Expression analysis of DMT genes using GeneChip ${ }^{\circledR}$}

GeneChip ${ }^{\circledR}$ data were from Tang et al [52]. Five developmental stages in anther length $(1.5 \mathrm{~mm}, 2.0 \mathrm{~mm}$, $2.2 \mathrm{~mm}, 2.5 \mathrm{~mm}$, and $3.0 \mathrm{~mm}$ ) refers to 10 time points from BS366, a wheat temperature-sensitive genic male sterile (TGMS) line under fertile and sterile conditions. Probe sets were considered differentially expressed with statistical significance when P-values were 0.05 , corresponding to the false discovery rate of $5 \%$. Gene annotations were obtained by Batch BLASTx (E-value <e-10) using the sequences of Affymetrix probe sets [52]. Hierarchical clusterings of all annotated genes and the expressed TaDMT genes were generated according to their relative transcript levels in 10 samples using the $R$ package 
pheatmap. The R package topGO were used to cluster the functional categories across the list of significantly enriched functional GO terms.

\section{Pollen iodine staining of wheat lines}

Over 240 anthers from each wheat line were used for pollen iodine staining, and pollen iodine staining was performed according to [60]: the mature anther was squeezed using small tweezers to release the pollen grains; (iii) then, 1 to 2 drops of $\mathrm{I} 2-\mathrm{KI}$ solution $[6.7 \%$ o $(\mathrm{g} / \mathrm{ml}) \mathrm{KI}$ and $3.3 \%$ o $(\mathrm{g} / \mathrm{ml}) \mathrm{I} 2]$ were added; finally, the images were obtained using an Olympus BX41 laboratory microscope (Olympus, Tokoyo, Japan).

\section{Abbreviations}

DMT, DNA methyltransferase; P/TGMS, photoperiod or/and temperature-sensitive genic male sterile; MET1, Methyltransferase 1; CMT, Chromomethylase; DRM, Domains Rearranged Methyltransferase; DNMT2, DNA methyltransferase 2; RdDM: RNA-directed DNA methylation; siRNAs, small interfering RNAs; DEGs, differently expressed genes; GO, Gene Ontology; TEs, transposable elements; BAH, Bromo Adjacent Homology.

\section{Declarations}

\section{Acknowledgments}

We thank Prof. Ligeng Ma (College of Life Sciences, Capital Normal University, and Beijing Key Laboratory of Plant Gene Resources and Biotechnology for Carbon Reduction and Environmental Improvement) for his valuable advice concerning our manuscript.

\section{Authors' contributions}

GSQ and ZCP designed the experiments; LD, FBE, LYJ, and GJ. performed most of the experiments; TYM, ZLP, SRW, CZB, ZFT, and PBS completed identification of iodine dyeing and pollen fertility; WYB and CXC investigated and provided phenotype data of hybrids; LD and FBE wrote the manuscript; GSQ, ZCP , and WAP supervised the project.

\section{Funding}

This work was financially supported by Beijing Academy of Agriculture and Forestry Sciences Program (grant no. KJCX20170421), Important Crops Genetically Modified New Germplasm Creation (KJCX20200205), and Beijing Postdoctoral Research Foundation (2020-22-109). The funding bodies didn't participate in the design of the study, collection, analysis, and interpretation of data or in writing the manuscript.

\section{Availability of data and materials}


All data generated or analyzed during this study are included in this published article and the supplementary information files.

Ethics approval and consent to participate

Not Applicable.

Consent for publication

Not Applicable.

Competing interests

The authors declare that they have no competing interests.

\section{References}

1. Sasaki E, Kawakatsu T, Ecker JR, Nordborg M: Common alleles of CMT2 and NRPE1 are major determinants of $\mathrm{CHH}$ methylation variation in Arabidopsis thaliana. PLoS Genet 2019, 15(12):e1008492.

2. Xie M, Hong C, Zhang B, Lowdon RF, Xing X, Li D, Zhou X, Lee HJ, Maire CL, Ligon KL et al: DNA hypomethylation within specific transposable element families associates with tissue-specific enhancer landscape. Nat Genet 2013, 45(7):836-841.

3. Ponger L, Li WH: Evolutionary diversification of DNA methyltransferases in eukaryotic genomes. $\mathrm{Mol}$ Biol Evol 2005, 22(4):1119-1128.

4. Chen Zhu SZ, Chengzhe Zhou, Lan Chen, Haifeng Fu, Xiaozhen Li, Yuling Lin, Zhongxiong Lai and Yuqiong Guo: Genome-wide investigation and transcriptional analysis of cytosine-5 DNA methyltransferase and DNA demethylase gene families in tea plant (Camellia sinensis) under abiotic stress and withering processing. Peer J 2020(8:e8432 DOI 10.7717/peerj.8432).

5. Law JA, Jacobsen SE: Establishing, maintaining and modifying DNA methylation patterns in plants and animals. Nat Rev Genet 2010, 11(3):204-220.

6. Zhang HM, Lang ZB, Zhu JK: Dynamics and function of DNA methylation in plants. Nat Rev Mol Cell Bio 2018, 19(8):489-506.

7. Yang J, Lior-Hoffmann L, Wang SL, Zhang YK, Broyde S: DNA cytosine methylation: structural and thermodynamic characterization of the epigenetic marking mechanism. Biochemistry-Us 2013, 52(16):2828-2838.

8. Jullien PE, Susaki D, Yelagandula R, Higashiyama T, Berger F: DNA Methylation Dynamics during Sexual Reproduction in Arabidopsis thaliana. Curr Biol 2012, 22(19):1825-1830.

9. Hu L, Li N, Zhang Z, Meng X, Dong Q, Xu C, Gong L, Liu B: CG hypomethylation leads to complex changes in DNA methylation and transpositional burst of diverse transposable elements in callus cultures of rice. Plant Journal 2020, 101(1):188-203. 
10. Wang PF, Gao C, Bian XT, Zhao SZ, Zhao CZ, Xia H, Song H, Hou L, Wan SB, Wang XJ: Genome-wide identification and comparative analysis of cytosine-5 DNA methyltransferase and demethylase families in wild and cultivated peanut. Front Plant Sci 2016, 7.

11. Zemach A, Kim MY, Hsieh PH, Coleman-Derr D, Eshed-Williams L, Thao K, Harmer SL, Zilberman D: The Arabidopsisnucleosome remodeler DDM1 allows DNA methyltransferases to access H1containing heterochromatin. Cel/ 2013, 153(1):193-205.

12. Stroud H, Do T, Du JM, Zhong XH, Feng SH, Johnson L, Patel DJ, Jacobsen SE: Non-CG methylation patterns shape the epigenetic landscape in Arabidopsis. Nat Struct Mol Bio/ 2014, 21(1):64-+.

13. Cokus SJ, Feng SH, Zhang XY, Chen ZG, Merriman B, Haudenschild CD, Pradhan S, Nelson SF, Pellegrini M, Jacobsen SE: Shotgun bisulphite sequencing of the Arabidopsis genome reveals DNA methylation patterning. Nature 2008, 452(7184):215-219.

14. Mathieu O, Reinders J, Caikovski M, Smathajitt C, Paszkowski J: Transgenerational stability of the Arabidopsis epigenome is coordinated by CG methylation. Cell 2007, 130(5):851-862.

15. Genger RK, Kovac KA, Dennis ES, Peacock WJ, Finnegan EJ: Multiple DNA methyltransferase genes in Arabidopsis thaliana. Plant Molecular Biology 1999, 41(2):269-278.

16. Hu LJ, Li N, Xu CM, Zhong SL, Lin XY, Yang JJ, Zhou TQ, Yuliang AZ, Wu Y, Chen YR et al: Mutation of a major CG methylase in rice causes genome-wide hypomethylation, dysregulated genome expression, and seedling lethality. P Natl Acad Sci USA 2014, 111(29):10642-10647.

17. Yamauchi T, Johzuka-Hisatomi Y, Fukada-Tanaka S, Terada R, Nakamura I, lida S: Homologous recombination-mediated knock-in targeting of the MET1a gene for a maintenance DNA methyltransferase reproducibly reveals dosage-dependent spatiotemporal gene expression in rice. Plant Journal 2009, 60(2):386-396.

18. Du J, Zhong X, Bernatavichute YV, Stroud H, Feng S, Caro E, Vashisht AA, Terragni J, Chin HG, Tu A et al: Dual binding of chromomethylase domains to H3K9me2-containing nucleosomes directs DNA methylation in plants. Cel/ 2012, 151(1):167-180.

19. Stroud H, Greenberg MVC, Feng SH, Bernatavichute YV, Jacobsen SE: Comprehensive analysis of silencing mutants reveals complex regulation of the Arabidopsismethylome. Cell 2013, 152(1-2):352364.

20. Haag JR, Pikaard CS: Multisubunit RNA polymerases IV and V: purveyors of non-coding RNA for plant gene silencing. Nat Rev Mol Cell Bio 2011, 12(8):483-492.

21. Matzke MA, Mosher RA: RNA-directed DNA methylation: an epigenetic pathway of increasing complexity. Nat Rev Genet 2014, 15(6):394-408.

22. Kawashima T, Berger F: Epigenetic reprogramming in plant sexual reproduction. Nat Rev Genet 2014, 15(9):613-624.

23. Martinez G, Kohler C: Role of small RNAs in epigenetic reprogramming during plant sexual reproduction. Curr Opin Plant Biol 2017, 36:22-28.

24. Gent JI, Ellis NA, Guo L, Harkess AE, Yao YY, Zhang XY, Dawe RK: CHH islands: de novo DNA methylation in near-gene chromatin regulation in maize. Genome Res 2013, 23(4):628-637. 
25. Cao X, Aufsatz W, Zilberman D, Mette MF, Huang MS, Matzke M, Jacobsen SE: Role of the DRM and CMT3 methyltransferases in RNA-directed DNA methylation. Curr Biol 2003, 13(24):2212-2217.

26. Moritoh S, Eun CH, Ono A, Asao H, Okano Y, Yamaguchi K, Shimatani Z, Koizumi A, Terada R: Targeted disruption of an orthologue of DOMAINS REARRANGED METHYLASE 2, OsDRM2, impairs the growth of rice plants by abnormal DNA methylation. Plant Journal 2012, 71(1):85-98.

27. Vieira GC, Vieira GF, Sinigaglia M, Valente VLD: Linking epigenetic function to electrostatics: The DNMT2 structural model example. PLoS One 2017, 12(6).

28. Jeltsch A, Ehrenhofer-Murray A, Jurkowski TP, Lyko F, Reuterd G, Ankri S, Nellen W, Schaefer M, Helm M: Mechanism and biological role of Dnmt2 in Nucleic Acid methylation. RNA Biol 2017, 14(9):11081123.

29. Goll MG, Kirpekar F, Maggert KA, Yoder JA, Hsieh CL, Zhang XY, Golic KG, Jacobsen SE, Bestor TH: Methylation of tRNAAsP by the DNA methyltransferase homolog Dnmt2. Science 2006, 311(5759):395-398.

30. Lin Z, Liu M, Damaris RN, Nyong'a TM, Cao D, Ou K, Yang P: Genome-wide DNA methylation profiling in the Lotus (Nelumbo nucifera) flower showing its contribution to the stamen petaloid. Plants (Basel) 2019, 8(5).

31. Xing MQ, Zhang YJ, Zhou SR, Hu WY, Wu XT, Ye YJ, Wu XX, Xiao YP, Li X, Xue HW: Global Analysis Reveals the Crucial Roles of DNA Methylation during Rice Seed Development. Plant Physiology 2015, 168(4):1417-U1554.

32. Zhang B, Tieman DM, Jiao C, Xu Y, Chen K, Fei Z, Giovannoni JJ, Klee HJ: Chilling-induced tomato flavor loss is associated with altered volatile synthesis and transient changes in DNA methylation. Proc Natl Acad Sci U S A 2016, 113(44):12580-12585.

33. Bharti P, Mahajan M, Vishwakarma AK, Bhardwaj J, Yadav SK: AtROS1 overexpression provides evidence for epigenetic regulation of genes encoding enzymes of flavonoid biosynthesis and antioxidant pathways during salt stress in transgenic tobacco. J Exp Bot 2015, 66(19):5959-5969.

34. Chen R, Li M, Zhang HY, Duan LJ, Sun XJ, Jiang QY, Zhang H, Hu Z: Continuous salt stress-induced long non-coding RNAs and DNA methylation patterns in soybean roots. BMC Genomics 2019, 20(1).

35. Sun LF, Miao XF, Cui J, Deng J, Wang X, Wang YF, Zhang YF, Gao SR, Yang KJ: Genome-wide highresolution mapping of DNA methylation identifies epigenetic variation across different salt stress in Maize (Zea mays L.). Euphytica 2018, 214(2).

36. Van Dooren TJM, Silveira AB, Gilbault E, Jimenez-Gomez JM, Martin A, Bach L, Tisne S, Quadrana L, Loudet $\mathrm{O}$, Colot $\mathrm{V}$ : Mild drought in the vegetative stage induces phenotypic, gene expression, and DNA methylation plasticity in Arabidopsis but no transgenerational effects. J Exp Bot 2020, 71(12):3588-3602.

37. Yap WS, Yeo YL, Lai KS, Wee CY: Epigenetic of DNA methylation patterns in lowland and upland malaysian rice cultivars under induced drought and salinity. New Biotechnol2016, 33:S167-S167.

38. Sicilia A, Scialo E, Puglisi I, Lo Piero AR: Anthocyanin Biosynthesis and DNA Methylation Dynamics in Sweet Orange Fruit [Citrus sinensis L. (Osbeck)] under Cold Stress. J Agr Food Chem 2020, 
68(26):7024-7031.

39. Shan XH, Wang XY, Yang G, Wu Y, Su SZ, Li SP, Liu HK, Yuan YP: Analysis of the DNA methylation of maize (Zea mays L.) in response to cold stress based on methylation-sensitive amplified polymorphisms. J Plant Biol 2013, 56(1):32-38.

40. Ebbs ML, Bender J: Locus-specific control of DNA methylation by the Arabidopsis SUVH5 histone methyltransferase. The Plant Cel/ 2006, 18(5):1166-1176.

41. Yelina NE, Choi K, Chelysheva L, Macaulay M, de Snoo B, Wijnker E, Miller N, Drouaud J, Grelon M, Copenhaver GP et al: Epigenetic remodeling of meiotic crossover frequency in Arabidopsis thaliana DNA methyltransferase mutants. Plos Genetics 2012, 8(8).

42. Teerawanichpan P, Chandrasekharan MB, Jiang YM, Narangajavana J, Hall TC: Characterization of two rice DNA methyltransferase genes and RNAi-mediated reactivation of a silenced transgene in rice callus. Planta 2004, 218(3):337-349.

43. Ahmad F, Huang X, Lan HX, Huma T, Bao YM, Huang J, Zhang HS: Comprehensive gene expression analysis of the DNA (cytosine-5) methyltransferase family in rice (Oryza sativa L.). Genet Mol Res 2014, 13(3):5159-5172.

44. Qian YX, Xi YL, Cheng BJ, Zhu SW: Genome-wide identification and expression profiling of DNA methyltransferase gene family in maize. Plant Cell Rep 2014, 33(10):1661-1672.

45. Steward N, Kusano T, Sano H: Expression of ZmMET1, a gene encoding a DNA methyltransferase from maize, is associated not only with DNA replication in actively proliferating cells, but also with altered DNA methylation status in cold-stressed quiescent cells. Nucleic Acids Res 2000, 28(17):3250-3259.

46. Dai Y, Ni ZF, Dai J, Zhao T, Sun QX: Isolation and expression analysis of genes encoding DNA methyltransferase in wheat (Triticum aestivum L.). Bba-Gene Struct Expr 2005, 1729(2):118-125.

47. Wang Z, Li J, Chen S, Heng Y, Chen Z, Yang J, Zhou K, Pei J, He H, Deng XW et al: Poaceae-specific MS1 encodes a phospholipid-binding protein for male fertility in bread wheat. Proc Natl Acad Sci U S A 2017, 114(47):12614-12619.

48. Shiferaw B, Smale M, Braun HJ, Duveiller E, Reynolds M, Muricho G: Crops that feed the world 10. Past successes and future challenges to the role played by wheat in global food security. Food Secur 2013, 5(3):291-317.

49. Schilling S, Kennedy A, Pan S, Jermiin LS, Melzer R: Genome-wide analysis of MIKC-type MADS-box genes in wheat: pervasive duplications, functional conservation and putative neofunctionalization. New Phytol 2020, 225(1):511-529.

50. Tucker EJ, Baumann U, Kouidri A, Suchecki R, Baes M, Garcia M, Okada T, Dong CM, Wu YZ, Sandhu A et al: Molecular identification of the wheat male fertility gene Ms1 and its prospects for hybrid breeding. Nat Commun 2017, 8.

51. Bai JF, Wang YK, Wang P, Duan WJ, Yuan SH, Sun H, Yuan GL, Ma JX, Wang N, Zhang FT et al: Uncovering male fertility transition responsive miRNA in a wheat photo-thermosensitive genic male sterile line by deep sequencing and degradome analysis. Front Plant Sci 2017, 8. 
52. Tang Z, Zhang L, Yang D, Zhao C, Zheng Y: Cold stress contributes to aberrant cytokinesis during male meiosis I in a wheat thermosensitive genic male sterile line. Plant Cell Environ 2011, 34(3):389405.

53. Li YF, Zhao CP, Zhang FT, Sun H, Sun DF: Fertility alteration in the photo-thermo-sensitive male sterile line BS20 of wheat (Triticum aestivum L.). Euphytica 2006, 151(2):207-213.

54. Zinn KE, Tunc-Ozdemir M, Harper JF: Temperature stress and plant sexual reproduction: uncovering the weakest links. J Exp Bot 2010, 61(7):1959-1968.

55. Min L, Li Y, Hu Q, Zhu L, Gao W, Wu Y, Ding Y, Liu S, Yang X, Zhang X: Sugar and auxin signaling pathways respond to high-temperature stress during anther development as revealed by transcript profiling analysis in cotton. Plant Physiol 2014, 164(3):1293-1308.

56. Ali A, Li Y, Chen H, Xu PZ, Zhang HY, Chen XQ, Liao YX, Fu SH, Wu TK, lqbal MZ et al: Effect of male sterile and fertile cytoplasm on nuclear DNA methylation in hybrid rice. Acta Physiol Plant 2019, 41(6).

57. Chen B, Zhang Y, Lu YL, Wang J, Zhang SZ, Lan H, Rong TZ, Cao MJ: DNA methylation analysis of sterile and fertile CMS-C hybrids and their parents in maize. J Plant Biochem Biot 2016, 25(1):3-11.

58. Ba QS, Zhang GS, Niu N, Ma SC, Wang JW: Cytoplasmic effects on DNA methylation between male sterile lines and the maintainer in wheat (Triticum aestivum L.). Gene 2014, 549(1):192-197.

59. Appels R, Eversole K, Feuillet C, Keller B, Rogers J, Stein N, Pozniak CJ, Stein N, Choulet F, Distelfeld A et al: Shifting the limits in wheat research and breeding using a fully annotated reference genome. Science 2018, 361(6403):661-+.

60. Li X, Gao S, Tang Y, Li L, Zhang F, Feng B, Fang Z, Ma L, Zhao C: Genome-wide identification and evolutionary analyses of bZIP transcription factors in wheat and its relatives and expression profiles of anther development related TabZIP genes. BMC Genomics 2015, 16:976.

61. Wang A, Xia Q, Xie W, Datla R, Selvaraj G: The classical Ubisch bodies carry a sporophytically produced structural protein (RAFTIN) that is essential for pollen development. Proc Natl Acad Sci US A 2003, 100(24):14487-14492.

62. Brautigam K, Cronk Q: DNA methylation and the evolution of developmental complexity in plants. Front Plant Sci 2018, 9.

63. Lee SI, Kim NS: Transposable elements and genome size variations in plants. Genomics Inform 2014, 12(3):87-97.

64. Deniz O, Frost JM, Branco MR: Regulation of transposable elements by DNA modifications. Nat Rev Genet 2019, 20(7):432-432.

65. Freitag M, Selker EU: Controlling DNA methylation: many roads to one modification. Curr Opin Genet Dev 2005, 15(2):191-199.

66. Mbichi RW, Wang QF, Wan T: RNA directed DNA methylation and seed plant genome evolution. Plant Cell Rep 2020, 39(8):983-996. 
67. Wang RB, Lou J, Li J: A mobile restriction modification system consisting of methylases on the IncA/C plasmid. Mobile DNA-Uk 2019, 10.

68. Bewick AJ, Niederhuth CE, Ji L, Rohr NA, Griffin PT, Leebens-Mack J, Schmitz RJ: The evolution of CHROMOMETHYLASES and gene body DNA methylation in plants. Genome Bio/ 2017, 18.

69. Yarychkivska O, Shahabuddin Z, Comfort N, Boulard M, Bestor TH: BAH domains and a histonelike motif in DNA methyltransferase 1 (DNMT1) regulate de novo and maintenance methylation in vivo. $J$ Biol Chem 2018, 293(50):19466-19475.

70. Sanchez DH, Paszkowski J: Heat-induced release of epigenetic silencing reveals the concealed role of an imprinted plant gene. Plos Genetics 2014, 10(11).

71. Kumar R, Chauhan PK, Khurana A: Identification and expression profiling of DNA methyltransferases during development and stress conditions in Solanaceae. Funct Integr Genomics 2016, 16(5):513528.

72. Kumar S, Stecher G, Li M, Knyaz C, Tamura K: MEGA X: molecular evolutionary genetics analysis across computing platforms. Mol Biol Evol 2018, 35(6):1547-1549.

73. Chen $\mathrm{C}$, Chen $\mathrm{H}$, Zhang Y, Thomas HR, Frank MH, He Y, Xia R: TBtools: an integrative toolkit developed for interactive analyses of big biological data. Mol Plant 2020, 13(8):1194-1202.

74. Lescot M, Dehais P, Thijs G, Marchal K, Moreau Y, Van de Peer Y, Rouze P, Rombauts S: PlantCARE, a database of plant cis-acting regulatory elements and a portal to tools for in silico analysis of promoter sequences. Nucleic Acids Research 2002, 30(1):325-327.

75. Livak KJ, Schmittgen TD: Analysis of relative gene expression data using real-time quantitative PCR and the 2(-Delta Delta C(T)) Method. Methods 2001, 25(4):402-408.

\section{Figures}




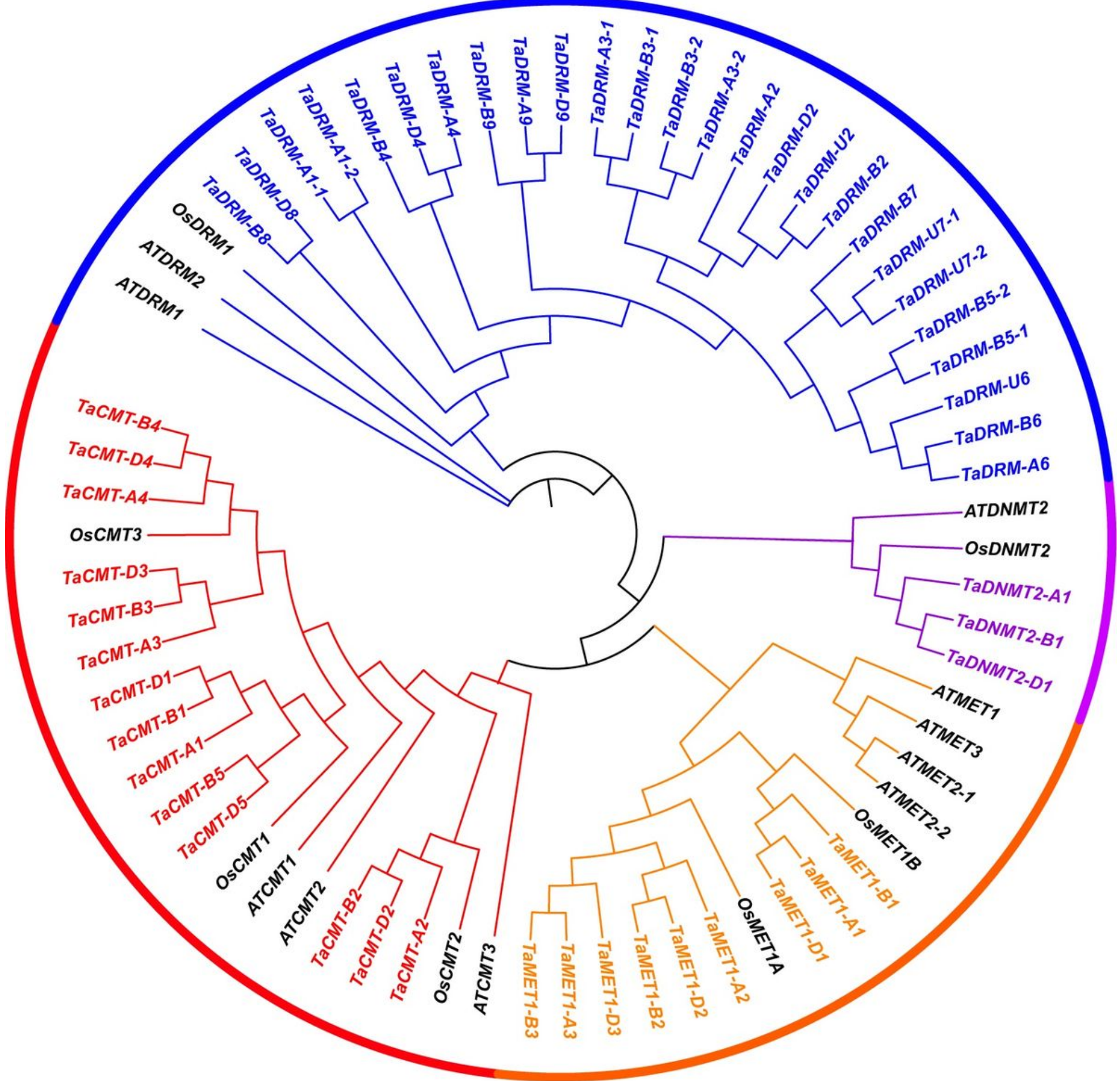

Figure 1

Phylogenetic analysis of DMT proteins from Arabidopsis, rice and wheat. A maximum likelihood phylogeny of DMT domain proteins Arabidopsis, rice, and wheat was generated using MEGA-X [72]. TaDMT genes are colored subclade-specific, whereas AtDMT genes and OsDMT genes are in black. 


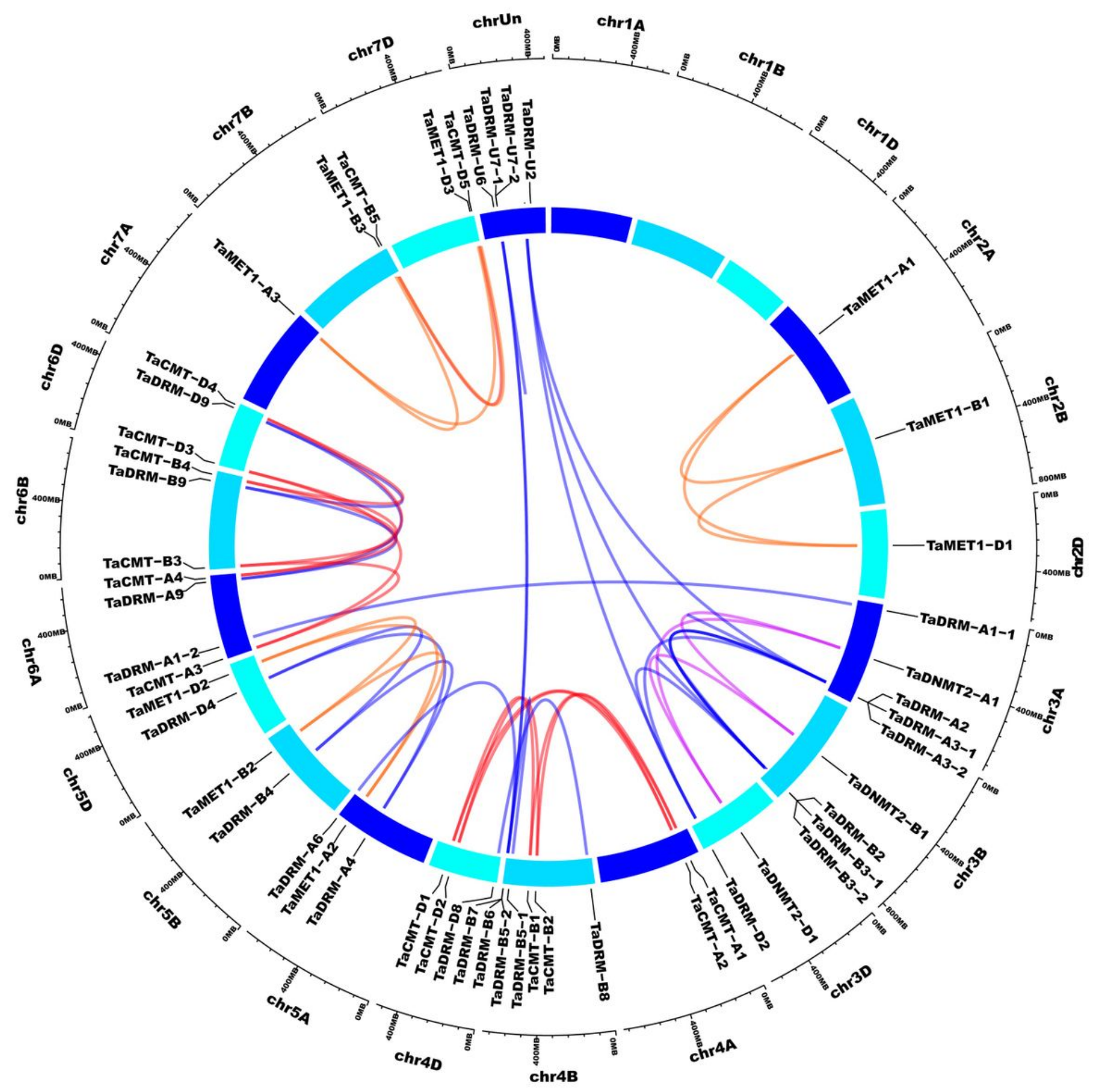

Figure 2

Chromosomal location and paralogs of DMT genes in wheat genome. All TaDMT genes were mapped to their respective locus in wheat genome in a circular diagram. Subgenomes are indicated by various shades of blue. Homeologous genes were inferred by phylogeny and linked with subfamily-specific colors as in Fig. 1 (inside of circle). 
A

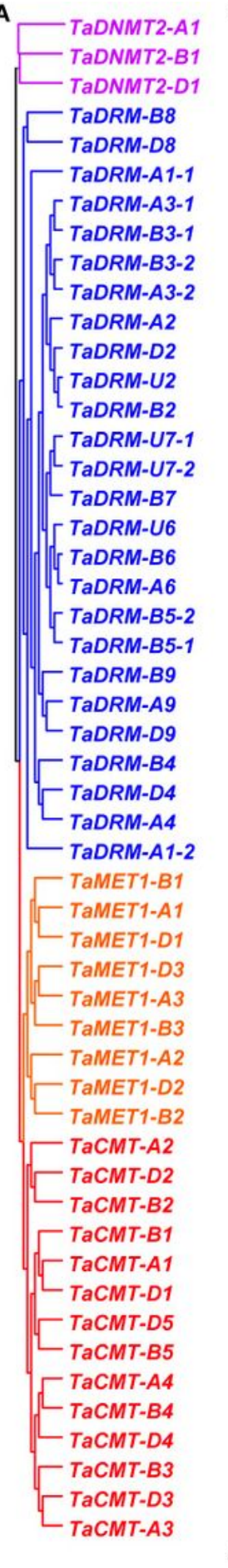

B

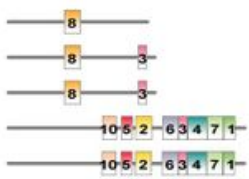

5.2-63471$108 \cdot 2-634771-$

$705.2-634.7 / 1-$

$7052-634771-$

$1082-63471-$

$1082-63471-$

$\longrightarrow 105 \cdot 2-63474-$

$708 \cdot 2-63477-$

$-108 \cdot 2-634747$

$\longrightarrow 05.2-63474-$

$105: 2-63477-$

$-108.2-63477-$

$705 \cdot 2-634771-$

$\longrightarrow 05 \cdot 2-634771-$

$\rightarrow 0$ - $2-634717-$

$-3474-$

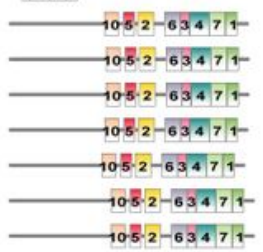

63471

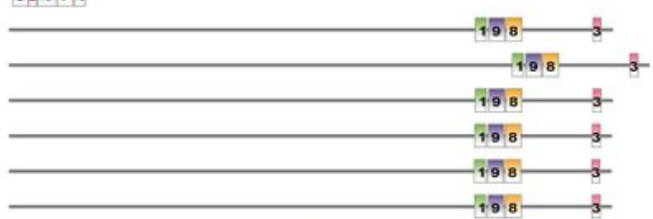

$-19-8-3-$
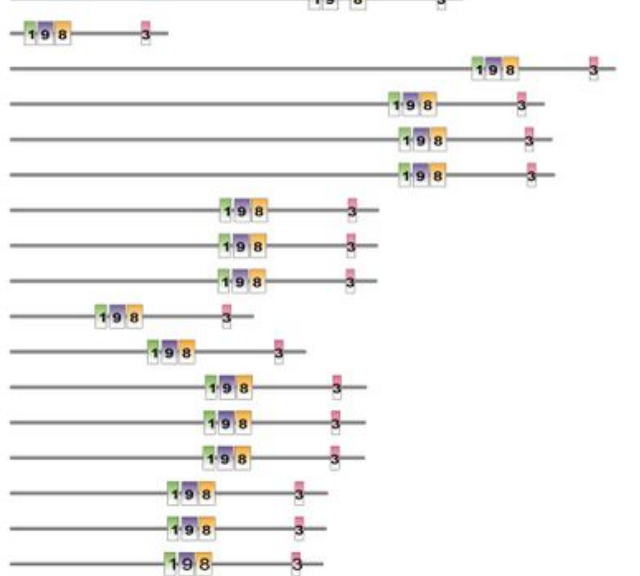

C

H-

Н-НH-НННН

H-HH-HНHН

$-\mathrm{HH}-\mathrm{H}$

H

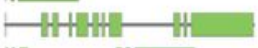

$H$

HLC

$+-\mathrm{H}-\mathrm{H}$

$\longrightarrow$

H

H:

$\longrightarrow$ HHH

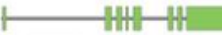

H

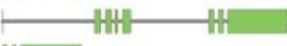

H.

H

TH

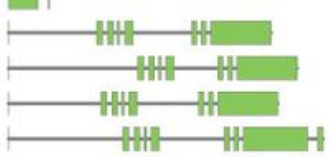

H

$\mathrm{H}$

H

-
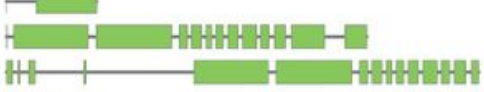

$\longmapsto \vdash+\mathrm{H}$

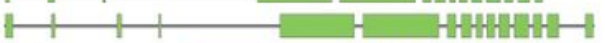

$\longrightarrow+$ HHHH

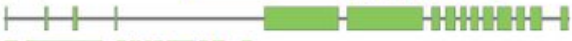

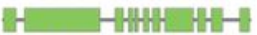

배ㅂㅐㅐㅐ는

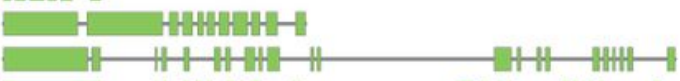

(H-HHH-H

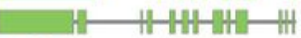

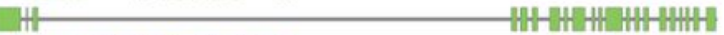

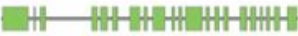

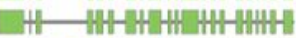

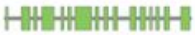

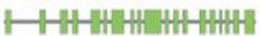

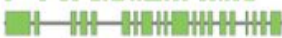

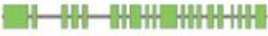

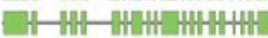

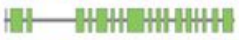

1 H

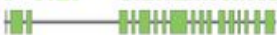

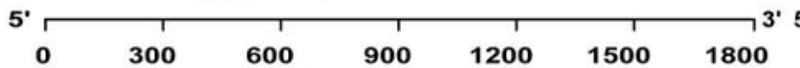

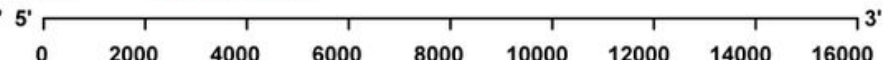

\begin{tabular}{l|l|l|l|l|l|l|} 
Motif 2 & Motif 3 & Motif 4 & Motif 5 & Motif 6
\end{tabular}

\begin{tabular}{|l|l|l|l|l|l|}
\hline Motif 7 & Motif 8 & Motif 9 & Motif 10 & Exon
\end{tabular}

\section{Figure 3}

Phylogenetic relationships (a), conserved motifs (b), and gene structures (c) of DMT genes in wheat. TaDMT genes are colored subclade-specific as in Fig. 1 
A

cellular nitrogen compound metabolic process cellular aromatic compound metabolic process. heterocycle metabolic process nucleobase-containing compound metabolic processnucleic acid metabolic process gene expression single-organism developmental process cellular component organization or biogenesiscellular component organizationorganelle organization negative regulation of biological processDNA metabolic process chromosome organization cell cyclecell cycle process cellular response to DNA damage stimulusDNA repairDNA methylationorganelle fissionDNA replication-

response to stimulus single-organism cellular processsingle-organism metabolic process response to abiotic stimulus small molecule metabolic process single-organism biosynthetic process organic acid metabolic processoxoacid metabolic process carboxylic acid metabolic process carbohydrate metabolic process. response to radiationresponse to light stimulus generation of precursor metabolites and energy single-organism carbohydrate metabolic process photosynthesissingle-organism catabolic process photosynthesis, light reactioncoenzyme metabolic process ribose phosphate metabolic process

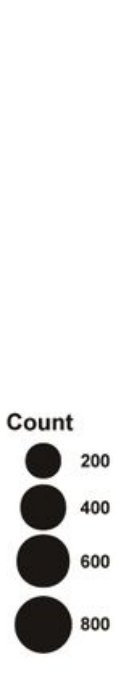

$-\log 10(P)$

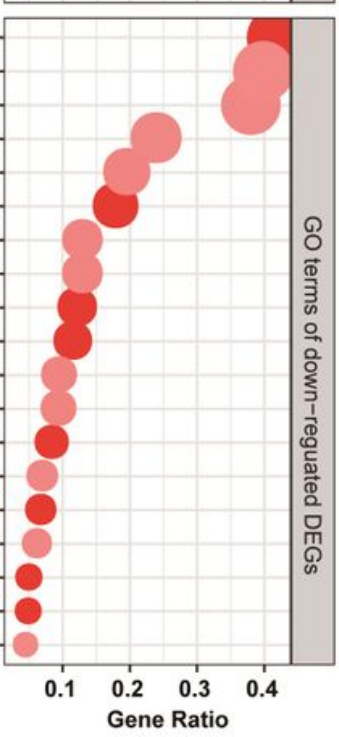

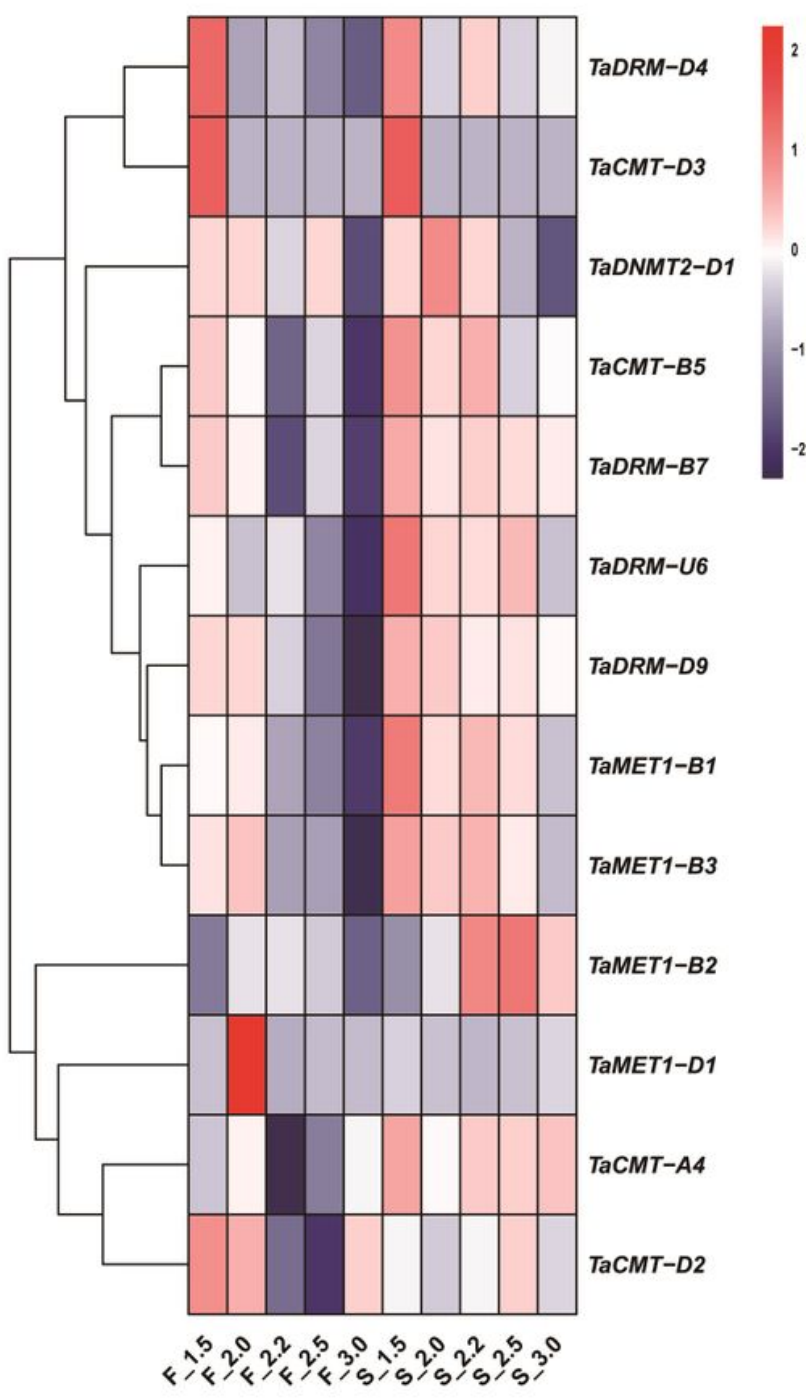

\section{Figure 4}

Enriched analysis of differentially expressed genes and expressed transcripts of TaDMT genes under sterile and fertile conditions in BS366 based on the microarray data. a The enriched biological process GO terms using differentially expressed genes in BS366. Significant enrichment is indicated by -log10 ( $P$ value). The above box indicates $\mathrm{GO}$ terms of up-regulated expressed genes; the below box indicates $\mathrm{GO}$ terms of down-regulated expressed genes. $b$ The hierarchical clustering of expressed TaDMT genes according to their relative transcript levels in 10 samples. Four main clusters are indicated with colors. The letter " $\mathrm{S}$ " indicates sterile treatment; " $\mathrm{F}$ " indicates fertile treatment. The numbers at the bottom of each panel $(1.5,2.0,2.2,2.5$, and 3.0$)$ represent the lengths of different anther samples. 

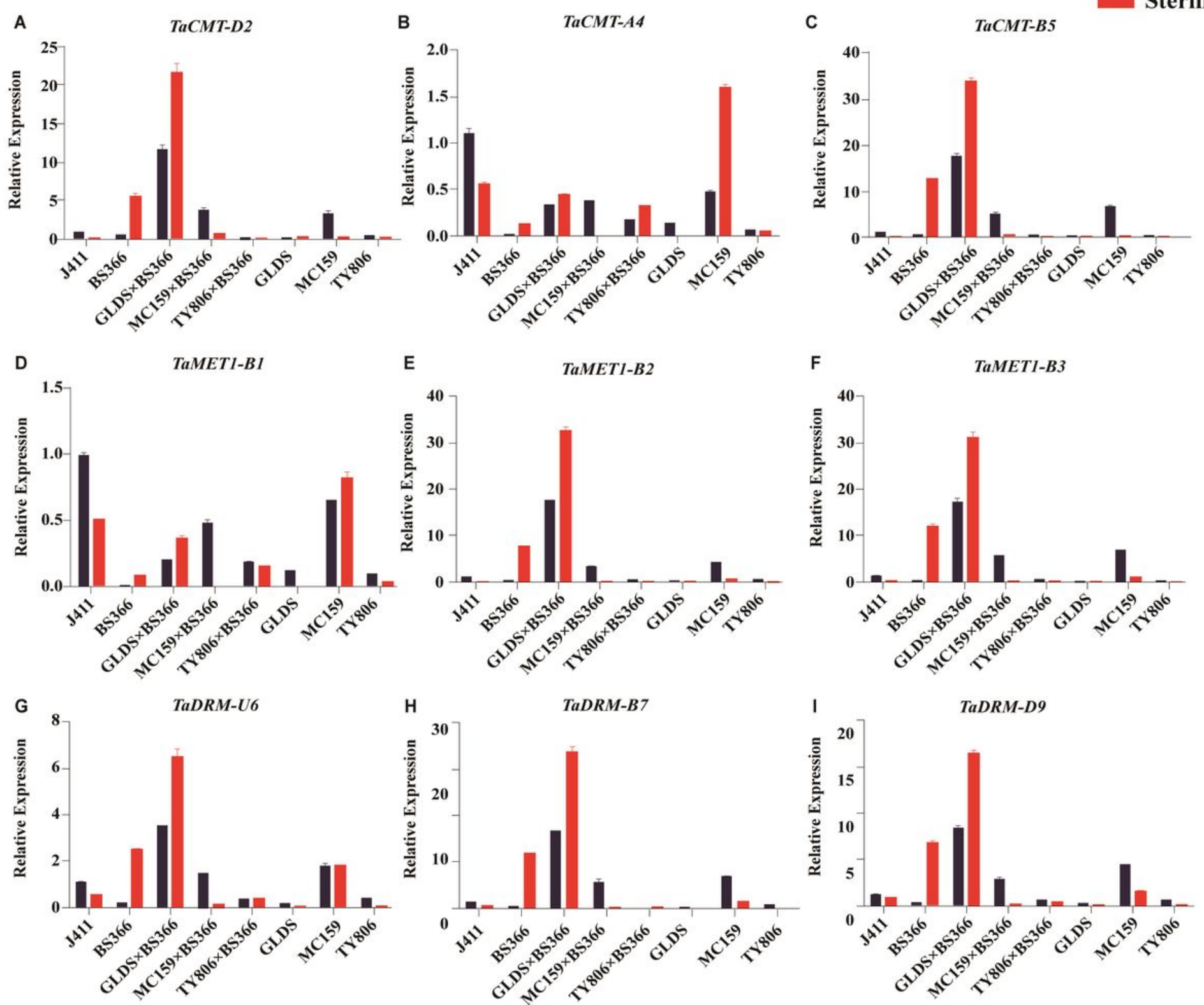

\section{Figure 5}

Transcript abundance of TaDMT genes under sterile and fertile conditions in wheat. Expression profiles of the TaDMT genes in BS366, four fertile wheat lines and three hybrids by qRT-PCR; BS366 is a sterile line; J411, GLDS, MC159, and TY806 are fertile lines; GLDS×BS366, MC159×BS366, and TY806×BS366 are three different fertility-restored hybrids. Data were presented as mean \pm standard deviation (SD). All experiments were repeated at least three times. 

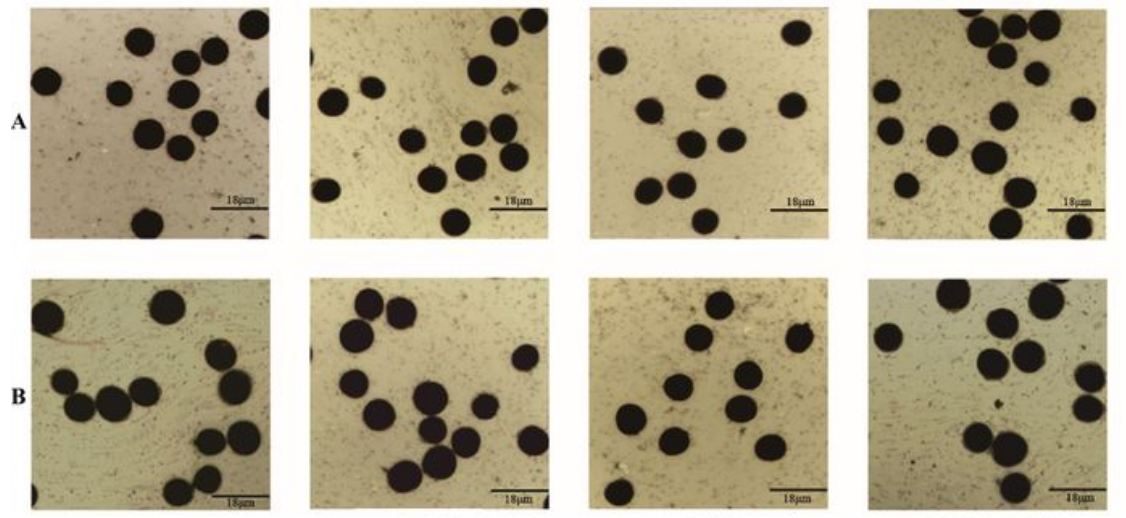

07YH91-43
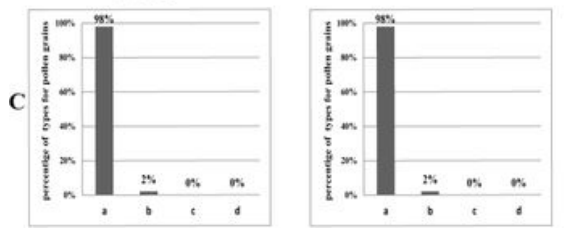

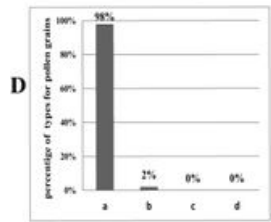
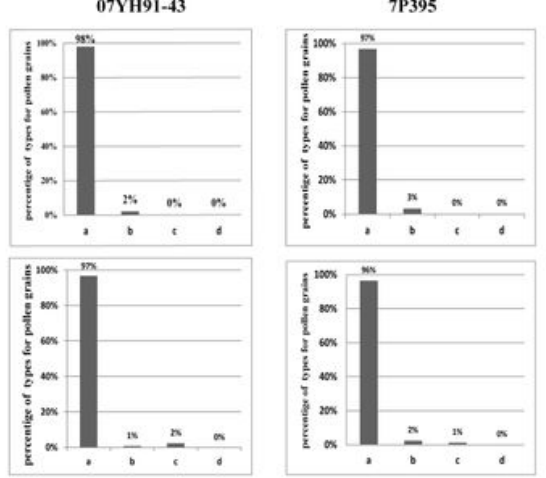

07GF83×BS366
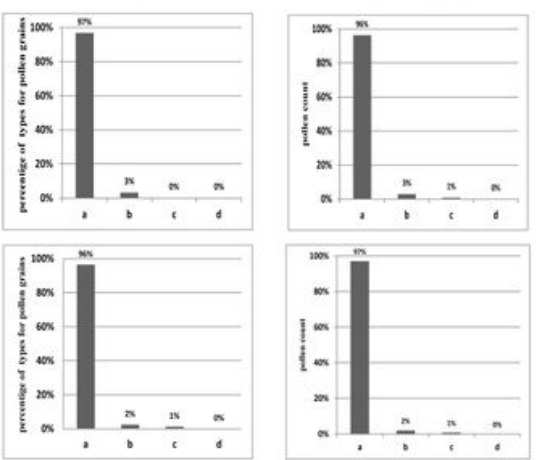
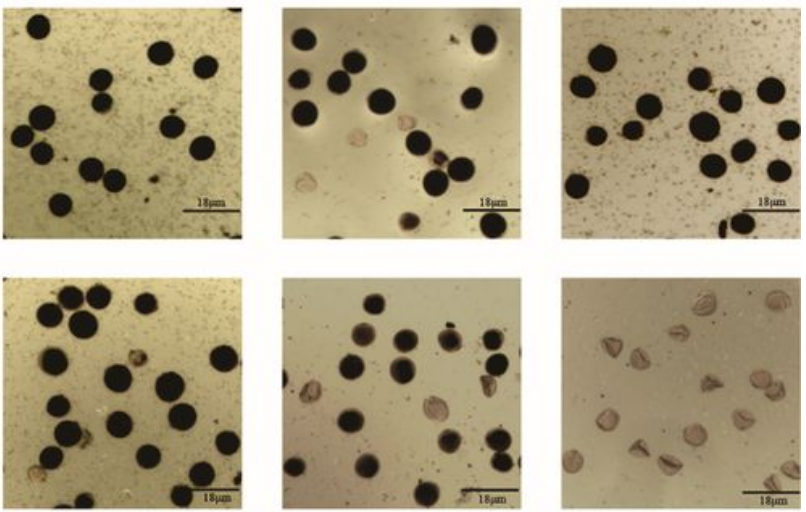

07YH91-43×BS366
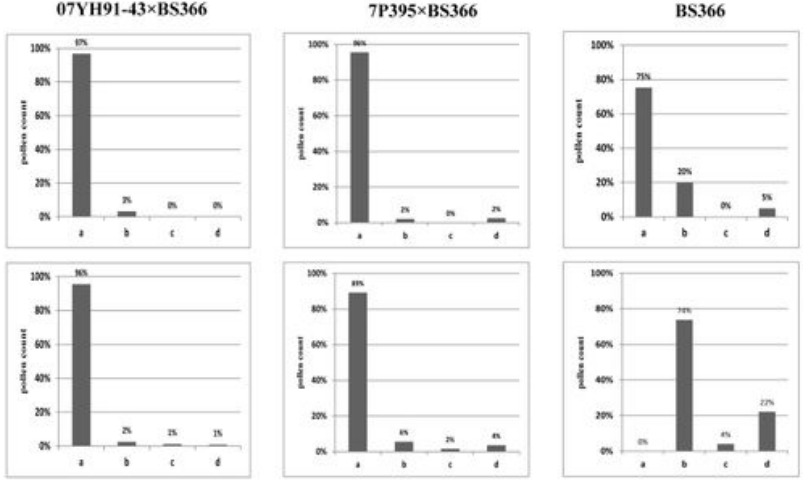

Figure 6

lodine staining of pollen grains and statistical analysis in different wheat lines. The wheat materials were taken separately from fertile $(a, c)$ and sterile $(b, d)$ conditions. a and b showed the iodine staining data; $c$ and $d$ showed the statistical analysis data. The total pollen counts used was over 240 in every line. a-d on the x-axis represent four different shape-color combinations or types, for the iodine-stained pollen grains:

(a) circular, opaque, and dark brown/black; (b) circular, opaque, or partially transparent and light brown/black; (c) circular, transparent, and light yellow; and (d) transparent with an irregular shape and light yellow. 
A
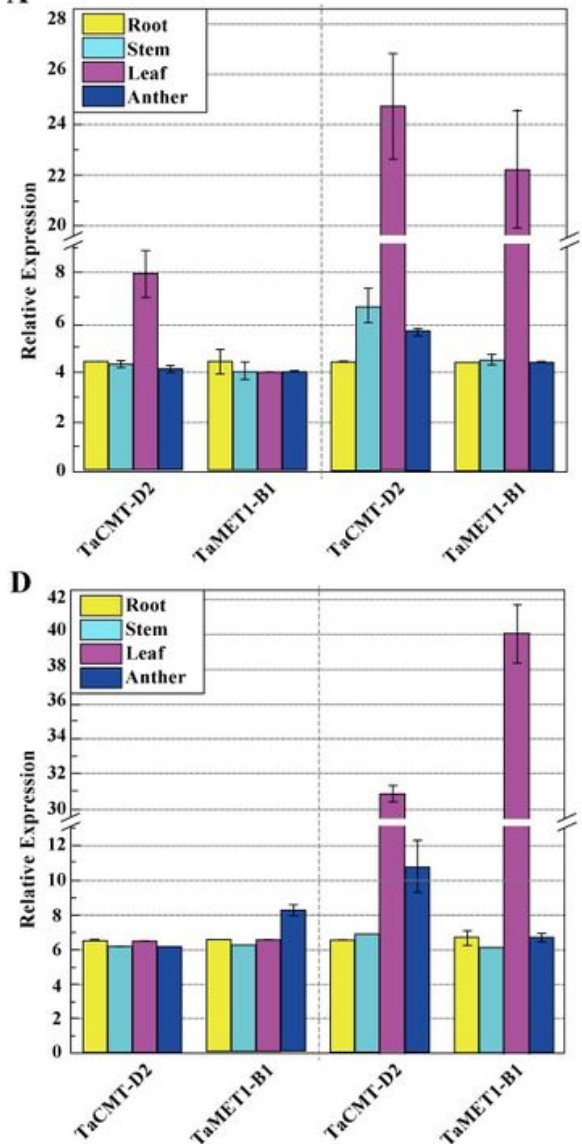

G

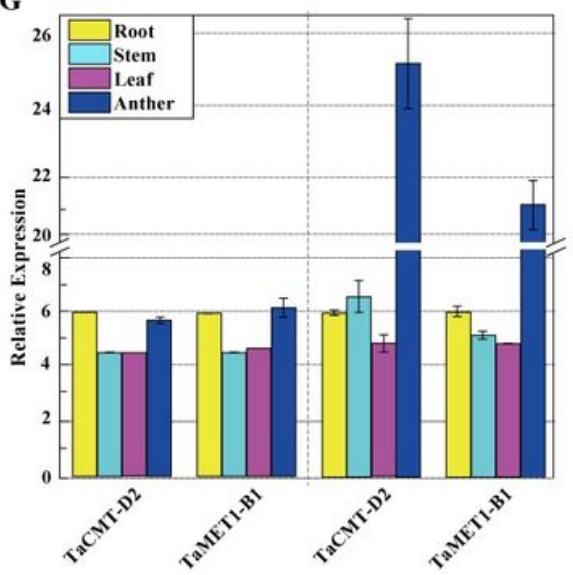

B
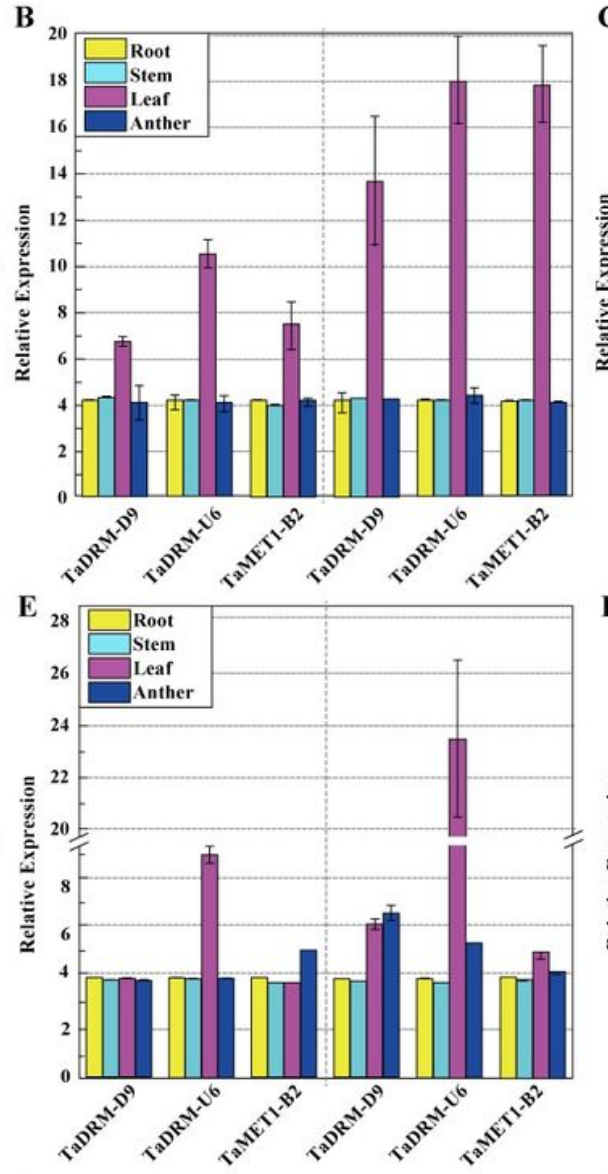

H

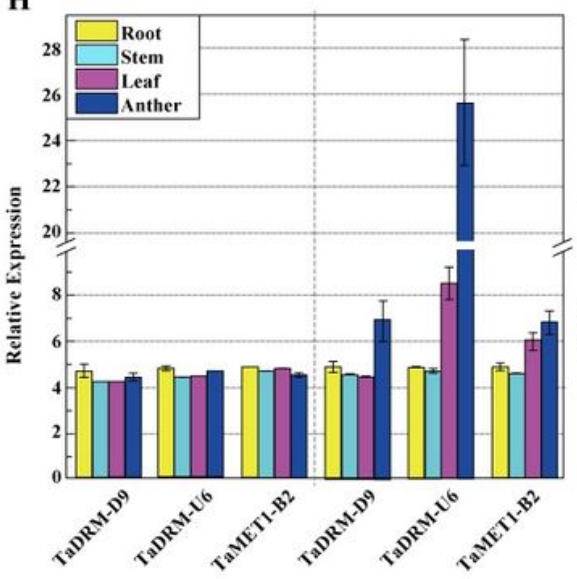

1
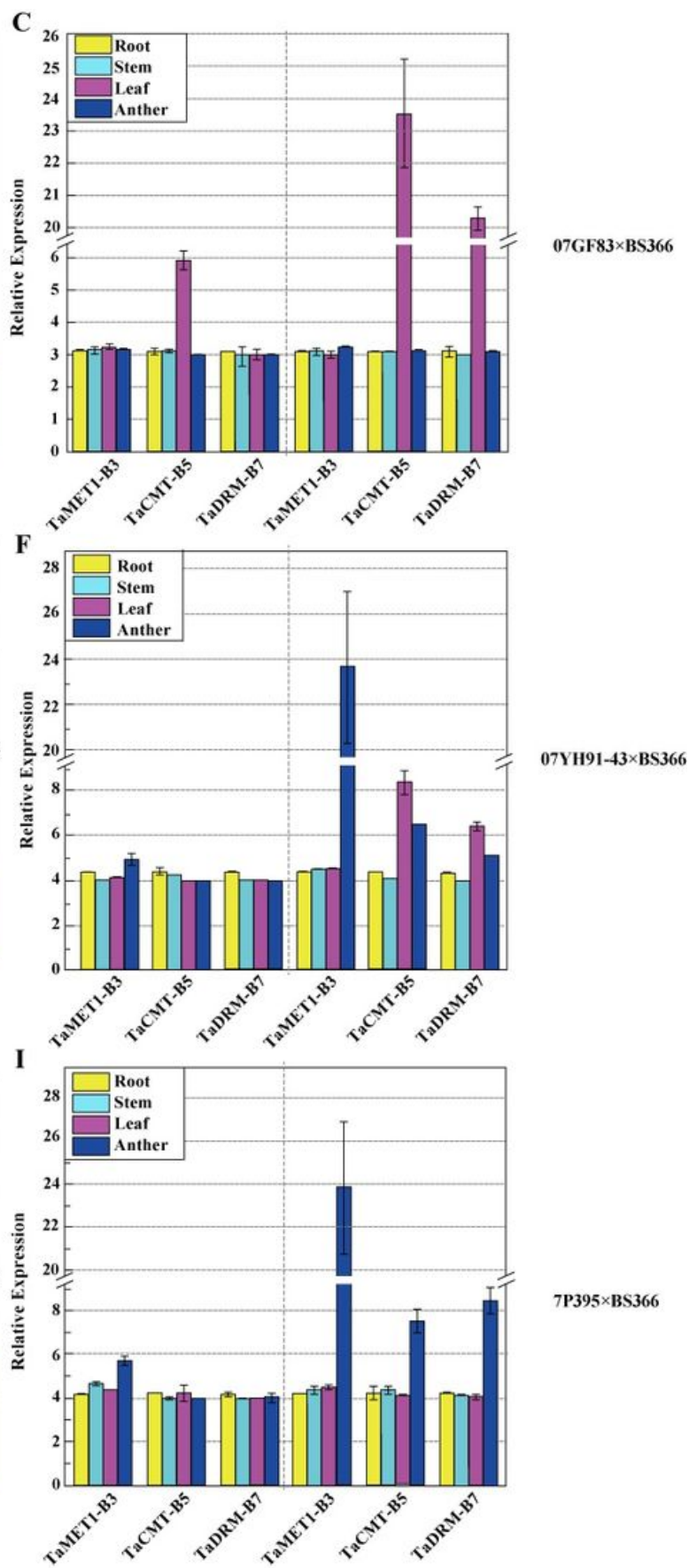

$7 \mathrm{P} 395 \times$ BS366

Figure 7

Tissue-specific expression profiles of TaDMT genes in different hybrids under fertile and sterile conditions. a-i show the expression levels of eight TaDMT genes under fertile (left) and sterile (right) conditions. 07GF83×BS366, 07YH91-43×BS366, and 7P395×BS366 are high-, medium-, and low- fertility F1 hybrids. Data were presented as mean \pm standard deviation (SD). All experiments were repeated at least three times. 


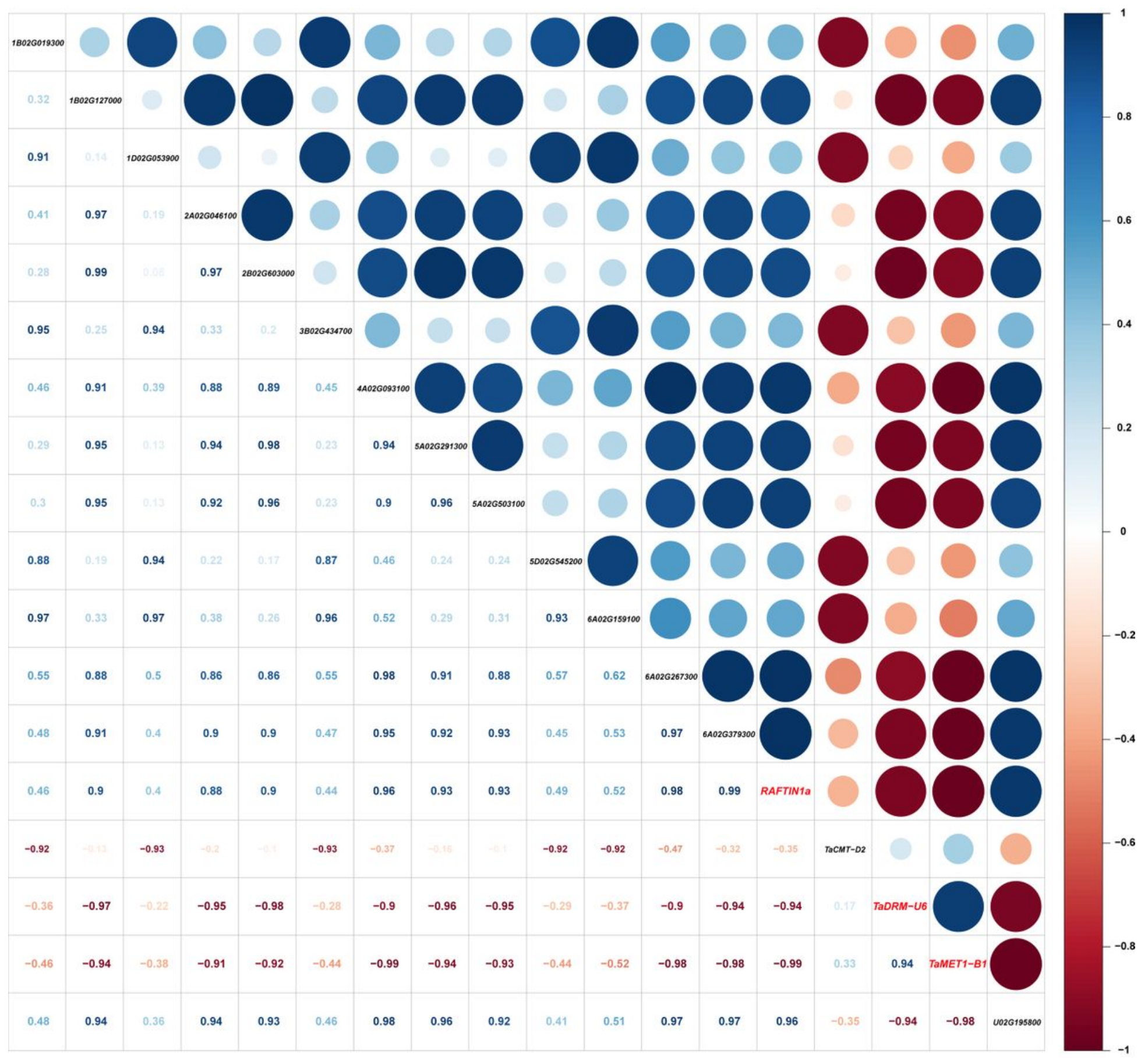

Figure 8

Expressional correlation analysis among candidate TaDMT genes and DEGs in BS366 under fertile and sterile conditions. The plots on the diagonal show the names of each gene. The values below the diagonal are Pearson correlation coefficients between genes, and the plots above the diagonal are circle plots of compared genes. The size of the circle represents the degree of correlation. Red values and circles indicate negative correlation; blue values and circles indicate positive correlation.

\section{Supplementary Files}


This is a list of supplementary files associated with this preprint. Click to download.

- SupplementalTables.xlsx

- SupplementalFiguresandLegends.docx 